\title{
A bi-objective model in sustainable dynamic cell formation problem with skill-based worker assignment
}

\author{
Farzad Niakan $^{\mathrm{a} 1}$, Armand Baboli ${ }^{\mathrm{a}}$, Thierry Moyaux ${ }^{\mathrm{a}}$, \\ Valérie Botta-Genoulaz ${ }^{\mathrm{a}}$ \\ a INSA-Lyon, DISP Laboratory EA4570, Villeurbanne, France
}

\begin{abstract}
The most recent revolution in industry (Industrial Revolution 4.0) requires increased flexibility, agility and efficiency in the use of production equipment. The Dynamic Cellular Manufacturing System (DCMS) is one of the best production systems to meet such requirements. In addition, the increasing importance of environmental and social issues, along with recent laws, is forcing manufacturers and managers to take account of sustainability when designing and configuring manufacturing systems. This paper proposes a new bi-objective mathematical model of the Dynamic Cell Formation Problem (DCFP), in which both the worker's assignment and environmental and social criteria are considered. The first objective in this model is to minimize both production and labor costs, and total waste (e.g., energy, chemical material, raw material, $\mathrm{CO} 2$ emissions, etc.). Social criteria are represented as constraints. Due to the NP-hardness of this problem, we propose a new resolution approach called NSGA II-MOSA, that merges an efficient hybrid meta-heuristic based on the Non-dominated Sorting Genetic Algorithm (NSGA-II), with Multi-Objective Simulated Annealing (MOSA). Randomlygenerated test problems demonstrate the performance of our algorithm.
\end{abstract}

Keywords: Dynamic Cell formation; Sustainability; Bi-objective optimization; Non-dominated Sorting Genetic Algorithm, Multi-Objective Simulated Annealing.

${ }^{1}$ Corresponding author. Tel: +33685765729

E-mail address: farzad.niakan@insa-lyon.fr (Farzad Niakan) 


\section{Introduction}

In order to remain competitive, manufacturers and producers have been forced to increase the productivity and flexibility of their manufacturing systems. Depending on the specification of production in an Industrial Revolution 4.0 environment [1], a production system must have a high degree of flexibility and agility to deal with product changes. The Dynamic Cellular Manufacturing System (DCMS) is one of the well-known production systems that meets this requirement. The Cellular Manufacturing System (CMS) based on Group Technology attempts to classify parts and machines in order to create cells and part-families; this is called a Cell Formation Problem (CFP). This classification is done according to the similarities in the geometry and operation process of each part, as well as a reduction of Work-In-Process (WIP) inventory, flow time and space utilization, while improving production planning and control. Most of the existing cell formation methods (static cell formation) have been developed for only one extended time period. But, under dynamic conditions, the components of product mixes change, the variety of products increases and the duration of product life cycles decreases. As a consequence, the configuration of the cells in CMS needs to be reorganized in order to maintain a high level of performance. Optimal cells in one period may therefore not be optimal in other periods because their configuration of part families and machine grouping may need either substitution of machines between cells or a change of the number of cells. These reasons motivate research on the configuration of Dynamic CMS (DCMS).

In previous investigations the economic aspect has traditionally been considered. Additionally, the pressures of communities, government and non-governmental organizations force managers and manufacturers to consider environmental and social criteria as well. In 1987 the United Nations World Commission on Environment and Development (WCED) [2] coined the now famous definition of sustainable development as a "development that meets the needs of the present without compromising the ability of future generations to meet their own needs". In this definition, the trade-off between present and future depends on how we choose to balance economic, social and environmental criteria. For these reasons, sustainability has recently attracted research. Despite the potential impact of sustainability on most decision-making, only few areas on tactical and operational decision levels have been addressed, such as aggregate production planning, and allocation and routing problems in supply chains. To the best of our knowledge there have been no studies on layout and cell formation.

In order to fill this gap, this research proposes a bi-objective mathematical model of DCFP, in which the environmental aspect is represented as the objective, and the social issues are represented as the constraints. A trade-off is thus made between some related costs as economic criteria, and production waste as environmental criteria, without compromising social needs. A restriction on the maximum daily noise exposure level is also considered for worker assignment as a social issue. As a resolution approach, a new efficient algorithm called NSGA II-MOSA that is a hybrid of the Non-dominated Sorting Genetic Algorithm (NSGA II) and Multi-Objective Simulated Annealing (MOSA) is designed.

The paper is organized as follows. The next section reviews the literature related to DCFP. Section 3 motivates this research. Section 4 describes the assumptions and problem formulation, and Section 5 the hybrid NSGA II-MOSA algorithm. The experimental result and a comparison between the proposed hybrid NSGA II-MOSA hybrid and a traditional application of NSGA II and MOSA are shown in Section 6. Finally, Section 7 ends on conclusions and future research directions. 


\section{Literature review}

This section gives an overview of the most prominent research on DCMS. Due to the large number of investigations in this area, we focus mainly on recent studies. First, Rheault et al. [3] introduced the concept of a dynamic environment in CFP. Schaller et al. [4] integrated CFP with inventory aspects, then showed the performance of their model on multiple heuristics and evaluated several alternative lower bounding methods. Chen and Cao [5] proposed a mathematical model for multi-period Cellular Manufacturing Systems (Dynamic CMS) minimizing the total cost, which includes: inter-cell material handling, inventory holding and the setting up of cells. They also developed a Tabu Search (TS) method to obtain good solutions and show the efficiency of their model. Next, these authors [6] generated a robust system configuration by integrating cell formation and part allocation. They also proposed a twostage TS to find the optimal or near optimal solutions. Tavakkoli-Moghaddam et al. [7] presented a nonlinear integer model of DCMS with machine capacity limitation, machine replication, inter-cell movements and production in batches. They used constant and variable costs as well as reconfiguration and inter-cell movement costs to formulate their objective function. Some of these authors [8] applied a Memetic Algorithm (MA) to solve their DCMS model. Defersha and Chen [9] formulated a comprehensive model containing dynamic cell configuration, alternative routings, lot splitting, sequence of operations and workload balancing. They also considered machine adjacency and cell size capacity as constraints. Moreover, Defersha and Chen [10] also proposed a two-phase GA-based heuristic to solve DCFP with alternative routings. Safaei et al. [11],[12] presented a DCMS mathematical model with uncertain circumstances, assuming fuzzy demand and fuzzy machine availability. They solved their mixed-integer programming model by developing fuzzy programmingbased aspects to determine optimal cell configuration with maximum satisfaction of the fuzzy objective and constraints. Safaei et al. [13] proposed a mixed-integer programming model in DCMS with batch inter/intra-cell material handling, sequence of operations, alternative process plans and machine replication. In this study, the authors minimized machine variable/constant costs, inter/intra-cell movements and reconfiguration costs as objective function. Defesha and Chen [14] integrated DCMS with production lot sizing in their minimization model with both production and quality-related (operation, set up, inventory, etc.) costs. They solved this model with a linear programming-embedded GA. Defersha and Chen [15] developed a parallel GA approach for DCFP. Ahkioon et al. [16] formulated a mixed integer mathematical model in DCMS by considering routing flexibility. In other words, they made a trade-off between increased flexibility and the imposed additional cost of part routings. Aryanezhad et al. [17] proposed a model to combine Simultaneous Dynamic Cell formation with a Worker assignment Problem (SDCWP). The objective function in their model has two components: (i) production costs such as inter-cell material handling and machine costs in the planning horizon, and (ii) human issues consisting of hiring, training, salary and firing costs. Egilmez et al. [18] addressed three stochastic skill-based manpower allocation models which allocate each worker to a manufacturing cell according to their performance. They also proposed a four-phased hierarchical robust optimization method to optimize manpower assignment and system production rates. SaidiMehrabad et al. [19] proposed a mathematical model to integrate production planning and worker assignment. This model minimizes the costs of maintenance and overheads, system reconfiguration, backorder and inventory holding, training, and salaries. Safaei and Tavakkoli-Moghaddam [20] developed a mathematical model to integrate multi-period cell formation and subcontracting of production planning in DCMS. Their study makes a trade-off between production and outsourcing costs on the re-configuration of CMS. Bajestani et al. [21] formulated a multi-objective mathematical modeling in DCMS and minimized the sum of various costs and total cell load variation simultaneously. They obtained the Pareto-optimal frontier with a new multi-objective scatter search method. Wang et al. [22] presented a non-linear mixed integer problem in DCMS with three conflicting objectives 
(machine relocation cost, utilization rate of machine capacity, and total number of inter-cell movements over the entire planning horizon). Deljoo et al. [23] improved their previous model by correcting some essential errors which had reduced the model's efficiency.

Mahdavi et al. [24] proposed an integer non-linear program of DCMS, considering worker aspects such as worker assignment, alternative workers, available time of workers, hiring and firing costs, and workers' salaries. Furthermore, they utilized holding and backordering costs in their model as an inventory aspect to make it more realistic. Javadian et al. [25] presented a multi-objective problem of cellular manufacturing systems in a dynamic and deterministic production environment to simultaneously minimize total cell load variation and the sum of miscellaneous costs (machine costs, inter/intra-cellular material handling, backorder, inventory holding and subcontracting). NSGA-II was applied to obtain optimal the Pareto-frontier. Rafiee et al. [26] integrated DCMS and an inventory lot sizing problem into a comprehensive mathematical model that includes several design factors: machine procurement, cell reconfiguration, preventive and corrective maintenance, intra/inter-cell material handling, subcontracting, inventory and defective parts replacement costs, etc. Saxena and Jain [27] provided a mixed-integer nonlinear programming model to merge machine breakdown effects and DCMS by incorporating reliability modeling. Kia et al. [28] proposed a mixed-integer non-linear programming model for DCMS by integrating three major decisions into the design of a CMS (cell formation, group layout) and developed an efficient SA method to solve their model. The objective function minimizes the total costs of intra/inter-cell movement, machine relocation, machine procurement, machine overheads and machine processing. Moreover, Kia et al. [29] addressed a mixed integer model for multi-floor layout design in DCMS. They developed a GA to solve their model. Rafiei and Ghodsi [30] presented a bi-objective model in DCMS and focused on human-related aspects. Their proposed model seeks (i) to minimize various costs including machine procurement relocation, machine variables, inter/intra-cell movement, and overtime and worker shifting as a first objective; and (ii) to maximize worker utilization as second objective. They proposed a hybridization of the ant-colony optimization algorithm with GA to solve their model. Kia et al [31] proposed a mixed integer mathematical model to integrate some new features such as: (i) manufacturing cells with variable numbers and shapes, (ii) machine depot keeping idle machines, (iii) machines of unequal areas, and (iv) manufacturing cells with regular rectangular shapes set on the continuous shop floor and in the design and configuration of the DCMS. Majazi-Dalfard [32] developed a new nonlinear integer programming model and considered the effect of distance on material flow. He also developed a new method including SA embedded in branch-and-cut to solve his model. Shiyas and Madhusudanan Pillai [33] formulated a model making a trade-off between the heterogeneity of cells and the inter-cell moves as two conflicting objectives in CMS. They also developed a heuristic to assign parts, and integrated it into a GA. Deep and Singh [34] proposed a comprehensive mathematical model in the design of a DCMS, considering multiple process plans for parts, and alternative process routes for each plan. These authors proposed a genetic algorithm based on heuristics to cope with the complexity of a problem. Finally, Niakan et al. [35] developed a bi-objective mathematical model of this problem in order to capture the trade-off between the minimization of the total costs and the maximization of social issues. They also designed a Non-dominated Sorting Genetic Algorithm (NSGA-II) to solve it. The classification of the previous studies is demonstrated in Table 1.

\section{\{Please insert Table 1 here.\}}

As this literature review shows, the design of CMS has received considerable attention, but that of DCMS, especially with regard to social and environmental aspects, has been investigated far less. In this study, a new bi-objective mathematical model is developed in order to integrate classical DCMS 
configuration aspects (economic) with social and environmental factors (sustainability) in an attempt to fill this gap in the literature and to provide new directions for future research.

\section{Motivation}

The most classical models in CFP focus only on economic criteria and over one single period, without any modification in demand or new products in the following periods. These assumptions are not realistic, especially with regard to the last decade, because reconfiguration of cells from one period to another increasingly becomes a necessity. The proposed model allows these evolutions to be taken into account. Furthermore, considering economic criteria is no longer enough to satisfy industries' demands, and finding a trade-off between economic, social and environmental criteria is increasingly necessary.

The first objective is associated with economic criteria such as machine fixed and variable costs, inter/intra-cell movement costs, machine procurement, installation and relocation costs, and salaries, and hiring/firing and training costs.

In order to be closer to reality, we assume that several costs (hiring, firing, and training for each skill) can vary from one period to another. The model therefore determines the best period for training workers, which allows for more flexibility. It also provides for the measurement of job opportunities and skill upgrading by training, etc. This kind of measurement can be very important in cost optimization and in workers' motivation.

In view of the importance of environmental criteria, the second objective focuses on waste minimization in order to take into account several kinds of waste $\left(\mathrm{CO}_{2}\right.$ emissions, energy loss, raw material scrap, water pollution, etc.). We include all waste in an aggregated way into this model, thus enabling the addition of other kinds of waste. Regarding the complexity of the model, social aspects such as Daily Noise Dosage (DND) have been introduced as constraints and our model is able to add other social constraints.

Finally, this paper also provides a contribution regarding the resolution approach. First, because of the complexity of our model, it has to be linearized. Second, due to the NP-hardness of design and configuration problems in DCMS, we develop a new hybrid meta-heuristic composed of two wellknown methods, viz., NSGA II and MOSA. Several demonstrations show the efficiency and performance of this hybrid meta-heuristic in comparison with the application of each of the two metaheuristics, separately.

\section{Problem description and formulation}

This section introduces our new bi-objective mathematical model of sustainable DCMS. As developed in the previous section, the first objective function minimizes various costs (Eq. 6) while the second one optimizes environmental aspects (Eq. 7).

\subsection{Environmental issue}

The industrial sector is considered to be one of the major sources of pollution in the world, and a large part of this pollution is produced in various ways such as material waste, water pollution, heat and Greenhouse Gas (GHG) emissions. Global warming, world pollution and ozone layer depletion force 
managers and manufacturers to improve the efficiency of their production systems, in order to decrease these forms of waste. This efficiency depends on the characteristics and age of the manufacturing equipment. The selection, configuration or reconfiguration of equipment are therefore one of the most important decision problems in reducing production waste. It is consequently necessary to consider environmental and social criteria in addition to traditional economic issues. Accordingly, several types of waste have been involved as a second objective function of our model in order to reflect the waste of each machine while processing every operation. This measurement can include various features, such as energy waste, chemical waste, raw material waste, GHG emissions, etc. Since the units of the considered features are different, several aggregation methods (e.g., normalized weighting method [36], AHP method [37], etc.) can be applied to transform these features into a single parameter called as machine waste for each operation. The waste for each operation of each machine can then be calculated as follows:

$$
w_{j, m}=\alpha_{1}\left(w_{\text {energy }}\right)+\alpha_{2}\left(w_{\text {chemical }}\right)+\alpha_{3}\left(w_{\text {raw material }}\right)+\alpha_{4}\left(w_{\text {GHG emission }}\right)
$$

where $\alpha_{i}$ are the normalized weights of each kind of waste. In the model that we propose, other environmental issues can be considered important in manufacturing systems. For example, the wasted energy (energy loss transformed into heat) for each operation on each machine may be estimated from the energy label (i.e., efficiency ranked A+, A, C, etc.) and power (i.e., energy consumption) provided by the manufacturer of this machine. This calculation is as follows:

$$
\text { power }=\frac{E}{t}
$$

Where $E$ is the amount of energy consumed to do the work and $t$ is the time taken, since we have:

$$
\begin{aligned}
& E=\text { useful energy }+ \text { energy loss } \\
& \text { Efficiency }=\frac{\text { useful energy }}{E}
\end{aligned}
$$

According to the above explanation, in this research an attempt has been made to choose some social and environmental aspects that are closely related to the DCMS configuration and have a valuable role in this problem. However, the proposed model can include new criteria and constraints, if necessary.

\subsection{Social issue}

Workers are continuously exposed to noise hazards in manufacturing environments in which many machines work simultaneously, generating a significant level of noise overall. According to Leigh and Miller [38], in their review of job-related illness data from the Bureau of Labor Statistics, hearing-loss accounts for more lost days than any other occupational illness and concerns more than 300 occupations. Danial et al. [39] found that workers' compensation claims in Washington State regarding annual disability settlements for hearing-related problems was close to $\$ 22.8$ million. From the point of view of human costs, occupational noise exposure not only causes social and psychological disorders for workers, but also these workers suffer from increased fatigue during shift work. Niebel and Freivalds [40] indicate that intermittent broadband noise can result in productivity decrease and greater employee fatigue, due to annoyance and distraction. 
Considerable effort has been devoted to controlling noise levels in workshops. Generally, there are various means to control industrial noise, such as proper design, maintenance, lubrication and alignment of machines, moving machines away from other workers, using barriers or shields in order to reflect high frequency noise, wearing hearing protection devices such as ear plugs or ear muffs, etc. Moreover, job reassignment and reduction of daily exposure times to high noise levels based on corresponding standards through dynamic cell formation, production planning and work force assignment can be applied in addition to the above-mentioned protective and preventive actions [41],[42]. Tharmmaphornphilsa et al. [43] provided a single-objective optimization model to minimize the maximum daily noise exposure among workers. They then ran a computer simulation model and compared the resulting schedules to those used in a sawmill. Aryanezhad et al. [44] proposed a multiobjective integer programming model to reduce the costs, noise exposure index and lower-back pain index for a single-period job-rotation schedule problem. They considered different skill levels as well as different job categories.

Therefore, regarding the noise problem, the worker assignment in the presented model is based on the maximum exposure to noise per day, which we call Daily Noise Dosage (DND). With regard to hearing loss, the National Institute of Occupational Safety and Health (NIOSH) [45] claimed that job rotation scheduling could be used as an effective means to control and reduce daily noise exposure. The Occupational Safety and Health Administration (OSHA) [46] as well as NIOSH [47] have provided threshold limit values for daily combined noise exposure levels, which represent conditions for permissible noise levels workers can be exposed to constantly.

\subsection{Problem assumptions}

The other assumptions of the model are explained in detail as follows:

1. Each part has an operation sequence and must be processed according to the sequence extracted from its route sheet.

2. The demand of each type of part in each period is known and constant.

3. The time capacity of each machine for processing parts is known and constant.

4. The worker assignment is done according to the worker's skill level and the machine's skill level category. As each machine type is categorized with a level, each machine level needs the worker to have the corresponding skill level. For example, the worker assigned to a level-2 machine must him- or herself have a skill level 2 at least (a worker with skill level 2 can work with a level-2 or a level-1 machine, but a worker with skill level 1 can work only with a level1 machine).

5. The workers at each skill level can be upgraded to the higher level by a training process which is modeled as a training cost.

6. The fixed cost of each machine is independent of the assigned workload. This cost is considered for each machine and includes the costs of total maintenance and overhead services.

7. The variable cost of each machine includes the operation cost and depends on the workload assigned to each machine.

8. The machine purchasing and selling costs are known and constant in each period. These costs include machine prices and freight charges, which means that removal costs between the place of storage and that of installation are not considered.

9. The relocation cost (un-installation, shifting and reinstallation) of each machine type between two periods is known and remains constant. 
10. Each machine type can process several operations, in other words, each processing of parts can be performed on different machine types with various operation times and costs.

11. The upper and lower bound of the cell size is known and remains fixed over the planning horizon.

12. Each period can be divided to several days and shifts.

13. The inter-intra movements of parts are performed in batches with different sizes and the related cost of this movement is dependent on the distance travelled. To decrease the complexity of the problem we assume that the distance between two cells (inter) is the same, and that for each cell the distance between two machines (intra) is the same. Furthermore, the dimensions of all machine types are equal. However, this assumption can be omitted if real inter- and intra-cell distances are known (for example by a table).

\section{$4.4 \quad$ Notations}

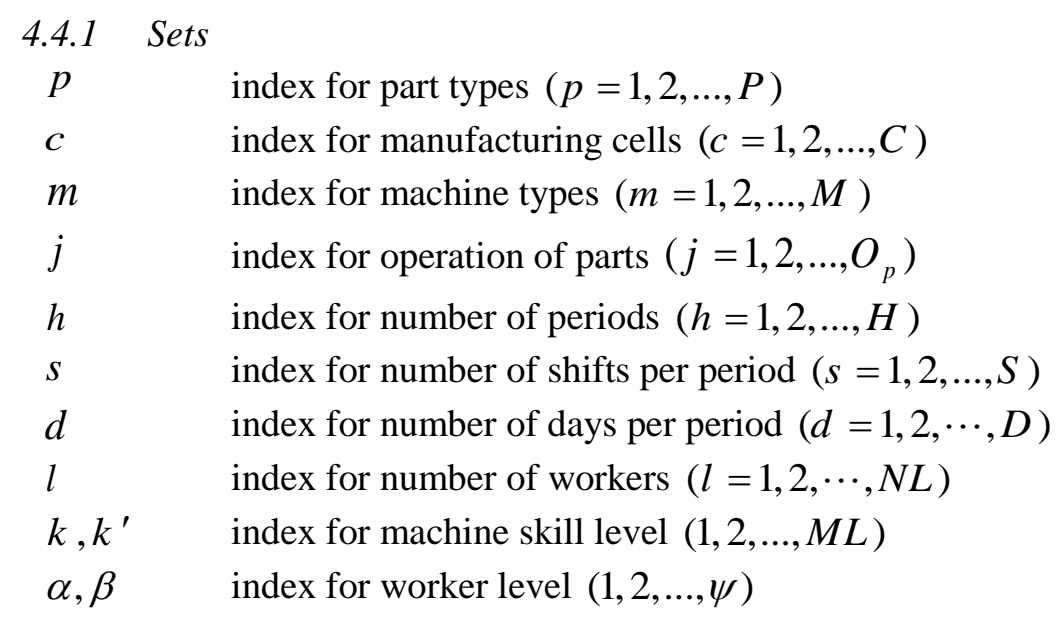

\subsubsection{Parameters}

$C \quad$ maximum number of cells

D number of days per period

$H \quad$ number of periods

$M \quad$ number of machine types

ML number of machine skill levels

$N L \quad$ number of workers

$P \quad$ number of parts

$O_{p} \quad$ number of operations for part type $p$

$S \quad$ number of shifts per period

$\psi \quad$ number of worker levels

$D_{p h} \quad$ demand for part $p$ in period $h$

$B_{p}^{\text {inter }} \quad$ batch size for inter-cell movement of part $p$

$B_{p}^{\text {intra }} \quad$ batch size for intra-cell movement of part $p$

$\gamma_{p}^{\text {inter }} \quad$ inter-cell movement cost per batch

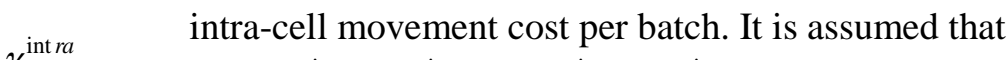

$\gamma_{p}^{\text {intra }} \quad \forall p\left(\gamma_{p}^{\text {intra }} / B_{p}^{\text {intra }}\right) \prec\left(\gamma_{p}^{\text {inter }} / B_{p}^{\text {inter }}\right)$ 
$\lambda_{m} \quad$ fixed cost of machine type $m$ per period

$\eta_{j, m} \quad$ variable cost of machine type $m$ to process operation $j$

$\delta_{m} \quad$ relocation cost of machine type $m$

$T_{m, h} \quad$ time-capacity of machine type $m$ in period $h$

UB maximum cell size

$L B \quad$ minimum cell size

$\varphi_{m} \quad$ cost of purchase of machine type $m$

$\omega_{m} \quad$ marginal revenue from selling machine type $m$

$\bar{L}_{K} \quad$ the combined noise level (dBA) measured at machine skill level $k$

$t_{j, p, m} \quad$ time required to process operation $j$ of part $p$ on machine type $m$

$a_{j, p, m} \quad 1$, if operation $j$ of part $p$ can be done on machine type $m ; 0$ otherwise

$w_{j, m} \quad$ total amount of waste of machine type $m$ to process operation $j$

$U G_{\alpha, \beta} \quad 1$, if training from skill level $\alpha$ to skill level $\beta$ is possible, 0 otherwise

$M T L_{m, k} \quad 1$, if machine of type $m$ belongs to machine skill level, 0 otherwise

$W P_{\alpha, k} \quad 1$, if a worker with skill level $\alpha$ can work on a machine with skill level $k ; 0$ otherwise

$h_{\alpha, h} \quad$ cost of hiring a worker with skill level $\alpha$ in period $h$

$C_{\alpha, \beta, h} \quad$ training cost of each $\alpha$-level worker for skill level $\beta$ in period $h$

$S_{\alpha, h} \quad$ salary of each $\alpha$-level worker in period $h$

$N L_{k} \quad$ number of workers needed for machine with skill level $k$

$F_{\alpha, h} \quad$ firing cost of each $\alpha$-level worker in period $h$

ALT available working time per worker in a working day

$t_{s} \quad$ time duration for each shift

$B N \quad$ an arbitrary big number

\subsubsection{Variables}

$N_{m, c, h} \quad$ number of type- $m$ machine assigned to cell $c$ at the beginning of period $h$

$K_{m, c, h}^{+} \quad$ number of type- $m$ machine added to cell $c$ at the beginning of period $h$

$K_{m, c, h}^{-} \quad$ number of type- $m$ machine removed to cell $c$ at the beginning of period $h$

$I_{m, h}^{+} \quad$ number of type- $m$ machine purchased at the beginning of period $h$

$I_{m, h}^{-} \quad$ number of machine type $m$ sold at the beginning of period $h$

$x_{j, p, m, c, h} \quad 1$, if operation $j$ of part $p$ is performed in cell $c$ in period $h$ by machine type $m ; 0$ otherwise

$x_{l, \alpha, k, h, s}^{\prime} \quad 1$, if worker $l$ with skill level $\alpha$ is assigned to a skill-level $k$ machine in period $h$ and shift $s ; 0$ otherwise

$E L_{\alpha, k, h} \quad$ number of existing workers with skill level $\alpha$ who are assigned to machine skill-level $k$ in period $h$

$H L_{\alpha, k, h} \quad$ number of hired workers with skill level $\alpha$ who are assigned to machine skill-level $k$ in period $h$ 
$F L_{\alpha, k, h} \quad$ number of fired workers in period $h$ with skill level $\alpha$ who were assigned to machine skill-level $k$

$T L_{\alpha, \beta, k, k^{\prime}, h} \quad$ number of $\alpha$-level workers who were working with a skill-level $k$ in period $h-1$, and are upgraded by training to higher skill level $\beta$ and are assigned to machine skill level $k^{\prime}$ in period $h$

$y_{\alpha, k^{\prime}, h} \quad 1$, if worker trains for level $\alpha$ skills and is assigned to machine skill-level $k^{\prime} ; 0$ otherwise

Note:

$$
E L_{\alpha, k, h}=\sum_{l=1}^{L} \sum_{s=1}^{S} x_{l, \alpha, k, h, s}^{\prime} \quad \forall \alpha, k, h
$$

\subsection{Mathematical formulation}

Once the sets, parameters and variables have been defined, the dynamic cell formation model with cost minimization, waste minimization and Daily Noise Dosage (DND) consideration is formulated as follows:

$$
\begin{aligned}
\text { Min } Z_{1}= & \sum_{h=1}^{H} \sum_{m=1}^{M} \sum_{c=1}^{C} \lambda_{m} \times N_{m, c, h}+\sum_{h=1}^{H} \sum_{m=1}^{M} \sum_{c=1}^{C} \sum_{p=1}^{P} \sum_{j=1}^{O_{p}} \eta_{j, m} \times D_{p h} \times t_{j, p, m} \times x_{j, p, m, c, h} \\
& +\frac{1}{2} \times \sum_{h=1}^{H} \sum_{p=1}^{P} \gamma_{p}^{\text {inter }} \times\left[\frac{D_{p h}}{B_{p}^{\text {inter }}}\right] \times \sum_{j=1}^{O_{p}-1} \sum_{c=1}^{C}\left|\sum_{m=1}^{M} x_{(j+1) p, m, c, h}-\sum_{m=1}^{M} x_{j, p, m, c, h}\right| \\
& +\frac{1}{2} \times \sum_{h=1}^{H} \sum_{p=1}^{P} \gamma_{p}^{\text {intra }} \times\left[\frac{D_{p h}}{B_{p}^{\text {intra }}}\right] \\
& \times \sum_{j=1}^{O_{p}-1} \sum_{c=1}^{C}\left(\sum_{m=1}^{M}\left|x_{(j+1) p, m, c, h}-x_{j, p, m, c, h}\right|-\left|\sum_{m=1}^{M} x_{(j+1) p, m, c, h}-\sum_{m=1}^{M} x_{j, p, m, c, h}\right|\right) \\
& +\frac{1}{2} \times \sum_{h=1}^{H} \sum_{m=1}^{M} \sum_{c=1}^{C} \delta_{m} \times\left(K_{m, c, h}^{+}+K_{m, c, h}^{-}\right)+\sum_{h=1}^{H} \sum_{m=1}^{M} I_{m, h}^{+} \times \varphi_{m}-\sum_{h=1}^{H} \sum_{m=1}^{M} I_{m, h}^{-} \times \omega_{m} \\
& +\sum_{h=1}^{H} \sum_{\alpha=1}^{\psi} \sum_{k=1}^{M L} h_{\alpha, h} \times H L_{\alpha, k, h}+\sum_{h=1}^{H} \sum_{\alpha=1}^{\psi} \sum_{\beta>\alpha}^{\psi} \sum_{k=1}^{M L} \sum_{k^{\prime}=1}^{M L} C_{\alpha, \beta, h} \times T L_{\alpha, \beta, k, k^{\prime}, h} \\
& +\sum_{h=1}^{H} \sum_{\alpha=1}^{\psi} \sum_{k=1}^{M L} S_{\alpha, h} \times\left(\sum_{l=1}^{L} \sum_{s=1}^{S} x_{l, \alpha, k, h, s}^{\prime}\right)+\sum_{h=1}^{H} \sum_{\alpha=1}^{\psi} \sum_{k=1}^{M L} F_{\alpha, h} \times F L_{\alpha, k, h}
\end{aligned}
$$

Min $Z_{2}=\sum_{h=1}^{H} \sum_{m=1}^{M} \sum_{c=1}^{C} \sum_{j=1}^{O_{p}} N_{m, c, h} \times w_{j, m} \times x_{j, p, m, c, h}$

Subject to:

$$
\begin{aligned}
& \sum_{c=1}^{C} \sum_{m=1}^{M} a_{j, p, m} \times x_{j, p, m, c, h}=1 \quad \forall j, p, h \\
& x_{j, p, m, c, h} \leq a_{j, p, m} \quad \forall j, p, c, m, h
\end{aligned}
$$




$$
\begin{aligned}
& \sum_{p=1}^{P} \sum_{j=1}^{O_{p}} D_{p, h} \times t_{j, p, m} \times x_{j, p, m, c, h} \leq T_{m, h} \times N_{m, c, h} \quad \forall m, c, h \\
& N_{m, c,(h-1)}+K_{m, c, h}^{+}-K_{m, c, h}^{-}=N_{m, c, h} \quad \forall m, c, h \\
& \sum_{c=1}^{C} N_{m, c,(h-1)}+I_{m, h}^{+}-I_{m, h}^{-}=\sum_{c=1}^{C} N_{m, c, h} \quad \forall m, h \\
& \sum_{m=1}^{M} N_{m, c, h} \leq U B \quad \forall c, h \\
& \sum_{m=1}^{M} N_{m, c, h} \geq L B \quad \forall c, h \\
& \sum_{k=1}^{M L} \sum_{\alpha=1}^{\psi} x_{l, \alpha, k, h, s}^{\prime} \leq 1 \quad \forall l, h, s \\
& \sum_{l=1}^{N L} \sum_{\alpha=1}^{\psi} x_{l, \alpha, k, h, s}^{\prime} \geq N L_{k} \quad \forall k, h, s \\
& x_{l, \alpha, k, h, s}^{\prime} \leq W P_{\alpha, k} \quad \forall l, \alpha, k, h, s \\
& \sum_{l=1}^{N L} \sum_{s=1}^{S} x_{l, \alpha, k, h, s}^{\prime}=\sum_{l=1}^{N L} \sum_{s=1}^{S} x_{l, \alpha, k,(h-1), s}^{\prime}+H L_{\alpha, k, h}-F L_{\alpha, k, h} \quad \forall \alpha, h, k \\
& +\sum_{v=1}^{\psi} \sum_{k^{\prime}=1}^{M L}\left(T L_{v, \alpha, k^{\prime}, k, h}-T L_{\alpha, v, k, k^{\prime}, h}\right) \\
& A L T \times \sum_{\alpha=1}^{\psi} \sum_{l=1}^{N L} \sum_{s=1}^{S} x_{l, \alpha, k, h, s}^{\prime} \geq \sum_{c=1}^{C} \sum_{m=1}^{M}\left(N_{m, c, h} \times T_{m, h} \times M T L_{m, k} \times N L_{k}\right) \quad \forall h, k \\
& F L_{\alpha, k, h} \leq B N \times W P_{\alpha, k} \quad \forall \alpha, k, h \\
& H L_{\alpha, k, h} \leq B N \times W P_{\alpha, k} \quad \forall \alpha, h, k \\
& T L_{\alpha, v, k, k^{\prime}, h} \leq B N \times W P_{\alpha, k} \quad \forall v, \alpha, k, k^{\prime}, h \\
& T L_{\alpha, v, k, k^{\prime}, h} \leq B N \times W P_{v, k^{\prime}} \quad \forall v, \alpha, k, k^{\prime}, h \\
& T L_{\alpha, v, k, k^{\prime}, h} \leq B N \times U G_{v, \alpha} \quad \forall v, \alpha, k, k^{\prime}, h \\
& \sum_{v>\alpha}^{\psi} \sum_{k^{\prime}=1}^{M L} T L_{\alpha, v, k, k^{\prime}, h}+F L_{\alpha, k, h} \leq E L_{\alpha, k,(h-1)} \quad \forall \alpha, k, h \\
& \sum_{v=1}^{\psi} \sum_{k=1}^{M L} T L_{v, \alpha, k, k^{\prime}, h} \leq B N \times y_{\alpha, k^{\prime}, h} \quad \forall \alpha, k^{\prime}, h \\
& F L_{\alpha, k^{\prime}, h} \leq B N \times\left(1-y_{\alpha, k^{\prime}, h}\right) \quad \forall \alpha, k^{\prime}, h \\
& 12.5 \times\left[\sum_{s=3 d-2}^{3 d} \sum_{k=1}^{M L} x_{l, \alpha, k, h, s}^{\prime} \times t_{s} \times 2^{\left(\frac{\bar{L}_{k}-90}{5}\right)}\right] \leq 100 \quad \forall l, \alpha, h, d
\end{aligned}
$$

In this mathematical model, an effort has been put into capturing trade-offs between economic and environmental factors, while the social aspects are considered as constraints. The first objective (Eq. 6) 
includes machine fixed cost, machine variable costs, inter-cell movement costs, intra-cell movement costs, machine relocation costs, purchasing and selling of machines, as well as hiring, training, salary and firing costs. The second objective function (Eq. 7) considers minimization of the total production waste amount resulting from machines. As noted in the preceding section, this amount is an aggregate value of several kinds of waste. Based on the precision level defined by decision-makers, several aggregation methods such as the normalized weighting method [36], the AHP method [37], etc. can be applied. To decrease the complexity of the model, social issues are considered as constraints (Eq. 28), and we focus on restriction of the maximum daily noise exposure level in workers' assignment to machines. The daily noise dose $D$ for the machine with exposure level $L$ (dBA) during period $t$ is calculated as follows

$$
D=12.5 \times t \times\left[2^{\frac{L-90}{5}}\right]
$$

According to the standard released by NIOSH [47] and OSHA [46], the combination of exposure level $\left(L_{k}\right)$ in duration of $t_{s}$ for each person should be less than $100 \mathrm{dBA}$.

Eqs. $(8,9)$ ensure that parts are processed according to plan and to required processes. The time capacity of planning periods is controlled by constraints in Eq. $(10)$. Eqs $(11,12)$ mainly consider the dynamic balance between consecutive periods in terms of, respectively, number of machines assigned to each cell, and number of machines purchased or sold (procurement cost) for each machine type. Eqs $(13,14)$ define the maximum number of machines per cell. Eq. (15) indicates that each worker has only one skill level and should be assigned to only one machine skill level. Constraint (16) ensures that enough workers are available for each skill level of machine at each period and shift. Constraint (17) applies the rules regarding the skill levels of workers who can work with machines with certain skill levels. Constraint (18) is the dynamic worker balance equation according to the hiring, firing and training of workers at each skill level and in each period. Constraint (18) guarantees that total demand in each period is satisfied by using hiring, firing or training. Constraint (19) ensures that sufficient numbers of workers are available to operate machines for each cell and machine type. Eqs. (20-24) ensure that firing, hiring and training are allowed when they are logically possible according to predefined rules (described in assumptions). The constraint in Eq. (25) ensures that the process of the firing the worker with skill-level $k$ and training of workers from level $k$ to a higher level in each period must be less than existed worker with the same level $(k)$ in previous period. Finally, Eqs. $(26,27)$ stipulate that firing newly-trained workers for skill level $\alpha$ is not allowed.

\subsection{Linearization}

The absolute part in the third and fourth terms of Eq. (6) make it nonlinear. Therefore, to transform it to the linear one for the third term, two non-negative variables $Z_{j p c h}^{1}$ and $Z_{j p c h}^{2}$ are introduced and the third term is rewritable as follows:

$$
\frac{1}{2} \times \sum_{h=1}^{H} \sum_{p=1}^{P} \gamma^{\text {inter }} \times\left\lceil\frac{D_{p h}}{B_{p}^{\text {inter }}}\right\rceil \times \sum_{j=1}^{O_{p}-1} \sum_{c=1}^{C}\left(z_{j p c h}^{1}+z_{j p c h}^{2}\right)
$$

The following constraint must be added to the proposed model.

$$
Z_{j p c h}^{1}-Z_{j p c h}^{2}=\sum_{m=1}^{M} x_{(j+1) p m c h}-\sum_{m=1}^{M} x_{j p m c h} \quad \forall j, p, c, h
$$


Similarly, to linearize the fourth term of the Eq. (6), $y_{j p m c h}^{1}$ and $y_{j p m c h}^{2}$, two non-negative variables, are introduced. The fourth term of the objective function can therefore be rewritten as follows:

$$
\frac{1}{2} \times \sum_{h=1}^{H} \sum_{p=1}^{P} \gamma^{\text {intra }} \times\left\lceil\frac{D_{p h}}{B_{p}^{\text {intra }}}\right\rceil \times \sum_{j=1}^{O_{p}-1} \sum_{c=1}^{C}\left(\sum_{m=1}^{M}\left(y_{j p m c h}^{1}+y_{j p m c h}^{2}\right)-\left(z_{j p c h}^{1}+z_{j p c h}^{1}\right)\right)
$$

And the following constraint must be added to the proposed model.

$$
y_{j p m c h}^{1}-y_{j p m c h}^{2}=x_{(j+1) p m c h}-x_{j p m c h} \quad \forall j, p, m, c, h
$$

Finally, the multiple integer and binary variables equation (8) made them nonlinear. It can be linearized as follows:

$$
\begin{array}{ll}
\text { Min } Z_{3}=\sum_{h=1}^{H} \sum_{m=1}^{M} \sum_{c=1}^{C} \sum_{j=1}^{O_{p}} N_{m, c, h} \times w_{j, m} & \\
\frac{X_{j, p, m, c, h}}{B N} \leq N_{m, c, h} \leq x_{j, p, m, c, h} \times B N & \forall j, p, m, c, h
\end{array}
$$

\section{Hybrid NSGA II-MOSA algorithm}

According to the previous studies, CFP has been mentioned as an NP-hard optimization problem [48], [30]. In order to cope with the complexity of the proposed mathematical model, a new hybrid of a non-dominated sorting genetic algorithm and a multi-objective simulated annealing (NSGA IIMOSA) is now proposed.

Non-dominated sorting genetic algorithm II (NSGA II) is an evolutionary algorithm commonly used in problems with more than one objective. With this method proposed by Deb et al. [49] we obtain a nearoptimal Pareto frontier. The mechanism of generation and changing of the population in NSGA II are the same as in GA. In fact, primary solutions are generated randomly and in each iteration new solutions are selected from the primary solutions (solution of previous iteration) and from solutions that are produced by crossover and mutation operations. In crossover operations, new solutions are produced by a combination of two parents that are selected by a binary tournament selection process. When in mutation, new solutions are generated by changing the characteristics of a member that is selected randomly. After theses operations, each solution is compared with others by two indicators: rank and crowding distance. The members of the population are sorted according to their rank: solutions in rank 1 are not dominated, and solutions in rank $R+1$ are only dominated by solutions in rank $R$. Since solutions with a same rank cannot dominate each other, they are compared by calculating their crowding distance, and a high value of crowding distance is preferred. Eq. (36) shows how to calculate the crowding distance $d_{i}$ of solution $i$ where $f_{i}{ }^{R} \quad$ represents the $R^{\text {th }}$ objective function value of the $i^{\text {th }}$ solution, and $f_{\min }^{R}$ and $f_{\max }^{R}$ the minimum and maximum value of the $R^{\text {th }}$ objective function, respectively. Furthermore, the crowding distance of the first and last points, the points which have optimum value in at least one objective, is assumed to be infinite.

$$
d_{i}=\sum_{R} \frac{f_{i+1}^{R}-f_{i-1}^{R}}{f_{\max }^{R}-f_{\min }^{R}}
$$

Next, the Pareto frontier ( $N$ solutions) obtained from NSGA-II is used as input for MOSA [50], which includes the concept of archives to trade off the solutions. Thus, in the proposed algorithm, we have two sets of solutions: representative solutions (RP) and archive of Pareto solutions (AP). These solutions are changed in each iteration and belong to representative solutions. Of course, in the first iteration of 
this algorithm, RP solutions are included in the Pareto solutions that are transmitted from NSGA-II, and the archive of Pareto solutions are sets of optimal Pareto solutions at the beginning of the algorithm. They are the same as RP.

In each iteration of MOSA, by applying a change operator, each RP solution will be changed due to the creation of neighboring solutions. Afterwards, this algorithm compares the new solution with the current one, and if the former is dominated by the latter, it will be added to the other solutions in AP as first rank. Otherwise, AP remains the same as in the previous iteration and also with $\alpha$ probability, the new solution is reported as RP or with probability $1-\alpha$, and the current solution is reported as RP. This procedure is repeated until the stopping criteria are satisfied (it=MaxIt). In each iteration of the proposed algorithm, an annealing schedule is selected to systematically decrease the temperature as the algorithm proceeds. As the temperature decreases, the algorithm reduces the extent of its search to converge to a minimum (see Fig 1). The mechanisms of decreasing temperature, $\alpha$ and MaxIt, are computed according to the following functions:

$$
\begin{aligned}
& T_{i t}=\beta \times T_{i t-1} \\
& T_{0}=C_{1}^{R P}+C_{2}^{R P} \\
& T_{f}=T_{0} \times 10^{-\gamma} \\
& \alpha=e^{-\left[\left(C_{1}^{\text {new }}+C_{2}^{\text {new }}\right)-\left(C_{1}^{R P}+C_{2}^{R P}\right)\right] / T_{i t}} \\
& \text { Maxit }=\left[\left(\log \left(\mathrm{T}_{f} / \mathrm{T}_{0}\right) / \log \beta\right)\right]
\end{aligned}
$$

Where $C_{1}$ and $C_{2}$ represent the values of first and second objective functions respectively, $\beta$ is the cooling rate, $T_{f}$ is the final temperature, $T_{0}$ is the initial temperature and $T_{i t}$ is the temperature in each iteration.

\{Please insert Fig. 1 here.\}

\subsection{Solution coding}

The structure of the solutions (Fig. 2) comprises 10 matrices as follows:

1. Matrix $[X]_{p \times r}^{h}$ consists of genes relating to the assignment of the part operations to the machines in period $h$ while $r$ is equal to $\max _{i}^{p}\left\{O_{i}\right\}$. The elements of the matrix $[X]$ are ascertained according to the $a_{j, p, m}$.

2. Matrix $[Y]_{p \times r}^{h}$ consists of genes signifying the assignment of part operations to the machines.

3. Matrix $[N]_{m \times c}^{h}$ consists of genes belonging to the number of available machines in each cell in period $h$ which is calculated as follows:

$$
N_{m, c, h}=\left[\frac{\sum_{p=1}^{P} \sum_{j=1}^{O_{p}} D_{p, h} \times t_{j, p, m} \times x_{j, p, m, c, h}}{T_{m}}\right] \quad \forall m, c, h
$$

The value of $N_{m, c, h}$ can be infeasible due to lower and upper bounds on the cell size. Therefore, an amendatory strategy is used to eliminate it. 
4. Matrix $[K]_{m \times c}^{h}$ includes the gene related to the machines moving into or out of each cell. It is noteworthy that the value of the elements in this matrix can be negative.

5. Matrix $[I]_{m \times c}^{h}$ consists of the gene signifying the number of machine procurement (selling and buying). The elements of this matrix can also be negative.

6. Matrix $[Z]_{l \times g}^{h}$ consists of genes relating to the assignment of the worker skill-level to the machine skill-level in period $h$, while $g$ is equal to $\max _{i \in l}\left\{\psi_{i}\right\}$. The elements of matrix [Z] are ascertained according to the $W P_{\alpha, k}$.

7. Matrix $[B]_{l \times g}^{h}$ consists of genes signifying the assignment of worker skill-level to the shifts. The element of matrix $[B]$ is generated by uniform distribution $\mathrm{U}(1, \mathrm{~s})$.

8. Matrix $[E]_{\alpha \times k}^{h}$ consists of genes which indicate the number of assigned workers with specific skill-levels to the specific machine skill-level in period $h$.

9. Matrix $[H]_{\alpha \times k}^{h}$ consists of genes which indicate the number of workers who are hired in period $h$.

10. Matrix $[F]_{\alpha \times k}^{h}$ consists of genes relating to the number of workers who are fired in period $h$.

\subsubsection{Crossover and Mutation Operator}

In this paper, the standard two-point crossover point is applied which are generated randomly. Each parent categorize to the three segment by crossover operator. The places of the middle segments are exchanged and produce the offspring. This procedure is demonstrated in Fig. 3.

Three different mutation methods are implemented as mutation operators; single mutation, multi mutation, and inversion mutation. For example, for the single mutation a part operation is selected randomly and assigned to the different machines that can be processed. The selected operation or the skill-level of the worker is chosen and assigned to another machine skill-level which can be assigned. Similarly, for the multi-mutation operator, a part is randomly picked by single mutation on all the operations. Finally, the inversion mutation is performed as an inversion of the sequence of cells which carry out the part operations.

\{Please insert Fig. 2 here.\}

\{Please insert Fig. 3 here.\}

\subsection{Parameter setting}

In order to increase the performance of our NSGA II-MOSA hybrid algorithm and to find the best results, we consider six vital parameters of NSGA II-MOSA, which should be carefully tuned:

- Number of members in a population (NPop)

- Number of iterations to find best results (MaxIt)

- Crossover rate (CrR)

- Mutation rate (MuR)

- Cooling rate (Beta)

- The power value to calculate $T_{f}$ (Gamma) 
As a result, a Taguchi method is used as a Design of Experiment (DOE) method to set the appropriate levels for the parameters of the algorithm. We thus define three levels for each parameter after an extensive analysis on proposed algorithm (Table 2).

\{Please insert Table 2 here.\}

In order to apply the Taguchi method, we consider three factors: Spacing Metric (SM), Distance to an ideal Point (D2P), and time. SM is a metric to define the quality of Pareto distribution in the objective space (uniformity). This metric is calculated by Eq. (43) as follows:

$$
S M=\frac{\sum_{i=1}^{N-1}\left|\bar{d}-d_{i}^{Q}\right|}{(N-1) \bar{d}}
$$

Where $d_{i}^{Q}$ represents the Euclidian distance between two consecutive points (i.e., points $i$ and $\left.i+1\right), \bar{d}$ is their average and $N$ is number of members in final non-dominated solutions.

Distance to an ideal point (D2P) is a metric that measures the average Euclidian distance between Pareto sets. An ideal point is defined by the decision maker. Finally, the computational time is another important metric to evaluate the performance of the algorithm. The Taguchi method arranges a matrix experiment with 27 trials (6 factors, 3 levels) according to the orthogonal array (Tables 3 and 4) and responses which are assigned to own trial. Regarding the same importance of three responses, normalization is used to convert all values to the same unit between 0 and 1 . Finally, evaluation of the Taguchi method leads to a figure (Fig. 4) as a graphic tool to show the single to noise ratio of each factor, in order to compare and select the best level for each factor. Analysis of the single to noise ratio shows that level 2 of NPop, i.e. 60, level 2 of MaxIt, i.e. 60, level 3 of $C r R$, i.e. 0.8, level 1 of $M u R$, i.e. 0.3, level 3 of Beta, i.e. 0.8 and level 3 of Gamma, i.e. 9 are better in comparison with other levels.

\{Please insert Table 3 here.\}

\{Please insert Table 4 here.\}

\{Please insert Fig. 4 here.\}

\section{Computational results}

In order to evaluate the performance and applicability of the proposed model and resolution approach, ten sets of samples (Table 5) are randomly generated based on the pattern given in Table 7. The complexity of test problems with regard to the number of variables and constraints is demonstrated in Table 6. The $\varepsilon$-constraint method is coded in optimization software GAMS 23.5 and hybrid NSGA IIMOSA, and MOSA and NSGA-II algorithms are developed using MATLAB R2012a software on a personal computer with Intel Core i5 CPU $2.27 \mathrm{GHz}$ and 4 GB RAM.

\{Please insert Table 5 here.\}

\{Please insert Table 6 here.\}

\{Please insert Table 7 here.\}

\subsection{Validation of correctness proposed approach and model}

To validate feasibility of proposed mathematical model and efficiency of the solution approach, a small numerical example (P1) is conducted and exact optimal Pareto frontier is illustrated in Table (8) using the well-known $\varepsilon$-constraint method. 


\title{
\{Please insert Table 8 here.\}
}

As Table (8) demonstrates, the Pareto frontier obtained by the proposed hybrid method is close to the $\varepsilon$-constraint, while the computational time $(T)$ for the hybrid method is almost 4 times smaller than the $\varepsilon$-constraint. ( $\left.T_{N S G A ~ I I-M O S A}=428, T_{\varepsilon \text {-constraint }}=1607\right)$.

\subsection{Experimental result}

The problems generated are then solved, first by using the NSGA-II and MOSA algorithms separately, and then with our hybrid NSGA II-MOSA. The Pareto optimal solutions obtained from the hybrid NSGA II-MOSA, NSGA II and MOSA for problems 3, 5, 7 and 10 are demonstrated in separate figures (Fig. 5, 6, 7 and 8). Through the optimal Pareto frontier obtained for problem 3, we also select three solutions (solution A: solution with best cost or first point of Pareto, solution B: a solution from middle of Pareto, solution C: solution with minimum waste or last point of Pareto). The cell configuration and workers pattern in the planning horizons of this example for selected solutions are depicted respectively in Figs 9, 10 and 11. Table 9 illustrates the part family assignment to manufacturing cells. To show the efficiency of NSGA II-MOSA as a hybrid method, the results (Paretooptimal frontiers) of each method are compared, using several metrics, as presented in the next section.

\author{
\{Please insert Fig. 5 here.\} \\ \{Please insert Fig. 6 here.\} \\ \{Please insert Fig. 7 here.\} \\ \{Please insert Fig. 8 here.\} \\ \{Please insert Fig. 9 here.\} \\ \{Please insert Fig. 10 here.\} \\ \{Please insert Fig. 11 here.\} \\ \{Please insert Table 9 here.\}
}

Considering the cell configuration on different solutions of problem 3, Figs 9, 10 and 11 illustrate that a large number of type 5 and 6 machines are relocated in the configuration of solution C (solution with minimum waste), which are more expensive but more efficient in decreasing waste. By contrast, in the configuration of solution A (solution with minimum cost), only one machine 5 is employed and there is no machine 6 on the planning horizon.

\subsection{Comparison Metrics}

To validate, compare and measure the performance of the proposed NSGA II-MOSA hybrid, three comparison metrics are considered (Table 10):

- Quality Metric (QM): This metric allows us to measure the ratio of non-dominated solutions obtained by each algorithm by putting together all their Pareto solutions. It reports the ratio of solutions related to each algorithm in final non-dominated solutions [21].

- Spacing Metric (SM): As mentioned in the previous section, this metric measures the uniformity of the spread of Pareto solutions, which is calculated by Eq. (43). The algorithm with a lower value of $S M$ is preferred [51].

- Diversification Metric (DM): This metric measures the spread of a Pareto solution set. The algorithm with a higher value of $D M$ is preferred [52]. Its function is defined as follows: 


$$
D M=\sqrt{\left(\frac{\max f_{i}{ }^{1}-\min f_{i}^{1}}{f_{\max }^{1, \text { total }}-f_{\min }^{1, \text { total }}}\right)^{2}+\left(\frac{\max f_{i}{ }^{2}-\min f_{i}{ }^{2}}{f_{\text {max }}^{2, \text { total }}-f_{\text {min }}^{2, \text { total }}}\right)^{2}}
$$

where $f_{\max }^{i, \text { total }}$ and $f_{\min }^{i, \text { total }}$ are the maximum and minimum values of each fitness function among all the dominated solutions obtained by the algorithms, respectively.

\section{\{Please insert Table 10 here.\} \\ \{Please insert Fig. 12 here.\} \\ \{Please insert Fig. 13 here.\} \\ \{Please insert Fig. 14 here.\}}

As shown in Table 7, Fig. 12, Fig. 13 and Fig. 14, the proposed hybrid NSGA II-MOSA has a better performance compared to NSGA II and MOSA taken separately. In other words, the hybrid NSGA IIMOSA hybrid algorithm can achieve the largest number of Pareto optimal solutions with better quality than NSGA II. Moreover, the average values of diversification and spacing metrics (Fig. 13 and Fig. 14) of our hybrid algorithm have greater and lower values, respectively, in comparison with NSGA II and MOSA taken separately (i.e., optimal Pareto solutions obtained by hybrid NSGA II-MOSA have more uniformity and diversity, respectively.)

\section{\{Please insert Fig. 15 here.\}}

Fig. 15 shows that the proposed hybrid of the NSGA II-MOSA algorithm takes only $50 \%$ more calculation time in average compared to NSGA II and MOSA. However, a part of this increase must be considered as warming up time (setup time), and this additional calculation time can be justified by a better quality solution.

\section{Conclusion and future research}

This paper addresses the issues of sustainable multi-period cell formation and worker assignment by considering economic, environmental and social aspects simultaneously - something that is largely overlooked in the literature on DCFP. A bi-objective mathematical model is developed, in which the first objective function minimizes the relevant costs of these issues, including machine and worker costs. The total production waste is minimized by the second objective function, and the maximum amount of Daily Noise Dosage (DND) for every worker is controlled by the constraint. In order to deal with the complexity of the problem and find a better solution, we develop a hybrid NSGA II-MOSA algorithm. We then compare its performance in terms of capability and reliability in several test problems with two conventional evolutionary algorithms (NSGA II and MOSA) on different metrics. The results demonstrate the supremacy of our hybrid algorithm over both NSGA II and MOSA.

For the extension of current research, other social and environmental issues can be considered, such as local job opportunities, job severity, occupational diseases, worker days lost due to injury, etc. Additionally, in order to be more realistic, various uncertain parameters and stochastic and possibilistic approaches, as well as political considerations, can be included in future research.

\section{Acknowledgment}

The authors gratefully acknowledge the scientific and financial support of ARC 8 of the Région Rhône-Alpes, France. 


\section{References}

[1] Brecher C, editor. Advances in Production Technology. Cham: Springer International Publishing; 2015. doi:10.1007/978-3-319-12304-2.

[2] Report of the World Commission on Environment and Development. Our Common Future. 1987.

[3] Rheault M, Drolet JR, Abdulnour G. Physically reconfigurable virtual cells: A dynamic model for a highly dynamic environment. Comput Ind Eng 1995;29:221-5.

[4] Schaller J, Selçuk Erengüç S, Vakharia A. A methodology for integrating cell formation and production planning in cellular manufacturing. Ann Oper Res 1998;77:1-21.

[5] Chen M, Cao D. Coordinating production planning in cellular manufacturing environment using Tabu search. Comput Ind Eng 2004;46:571-88.

[6] Cao D, Chen M. A robust cell formation approach for varying product demands. Int J Prod Res 2005;43:1587-605.

[7] Tavakkoli-Moghaddam R, Aryanezhad MB, Safaei N, Azaron A. Solving a dynamic cell formation problem using metaheuristics. Appl Math Comput 2005;170:761-80.

[8] Tavakkoli-moghaddam R, Safaei N, Babakhani M. Solving a Dynamic Cell Formation Problem with Machine Cost and Alternative Process Plan by Memetic Algorithms. Stoch. Algorithms Found. Appl., 2005, p. 213-27.

[9] Defersha FM, Chen M. A comprehensive mathematical model for the design of cellular manufacturing systems. Int J Prod Econ 2006;103:767-83.

[10] Defersha FM, Chen M. Machine cell formation using a mathematical model and a geneticalgorithm-based heuristic. Int J Prod Res 2006;44:2421-44.

[11] Safaei N, Saidi-Mehrabad M, Babakhani M. Designing cellular manufacturing systems under dynamic and uncertain conditions. J Intell Manuf 2007;18:383-99.

[12] Safaei N, Saidi mehrabad M, Tavakkoli moghaddam R. A fuzzy programming approach for a cell formation problem with dynamic and uncertain conditions. Fuzzy Sets Syst 2008;159:21536.

[13] Safaei N, Saidi mehrabad M, Jabal Ameli MS. A hybrid simulated annealing for solving an extended model of dynamic cellular manufacturing system. Eur J Oper Res 2008;185:563-92.

[14] Defersha FM, Chen M. A linear programming embedded genetic algorithm for an integrated cell formation and lot sizing considering product quality. Eur J Oper Res 2008;187:46-69.

[15] Defersha FM, Chen M. A parallel genetic algorithm for dynamic cell formation in cellular manufacturing systems. Int J Prod Res 2008;46:6389-413.

[16] Ahkioon S, Bulgak AA, Bektas T. Cellular manufacturing systems design with routing flexibility, machine procurement, production planning and dynamic system reconfiguration. Int J Prod Res 2008;47:1573-600. 
[17] Aryanezhad MB, Deljoo V, Mirzapour Al-e-hashem SMJ. Dynamic cell formation and the worker assignment problem: a new model. Int J Adv Manuf Technol 2009;41:329-42.

[18] Egilmez G, Erenay B, Süer GA. Stochastic skill-based manpower allocation in a cellular manufacturing system. J Manuf Syst 2014;33:578-88. doi:10.1016/j.jmsy.2014.05.005.

[19] Saidi-Mehrabad M, Paydar MM, Aalaei A. Production planning and worker training in dynamic manufacturing systems. J Manuf Syst 2013;32:308-14.

[20] Safaei N, Tavakkoli-Moghaddam R. Integrated multi-period cell formation and subcontracting production planning in dynamic cellular manufacturing systems. Int J Prod Econ 2009;120:30114.

[21] Aramoon Bajestani M, Rabbani M, Rahimi-Vahed AR, Baharian Khoshkhou G. A multiobjective scatter search for a dynamic cell formation problem. Comput Oper Res 2009;36:77794.

[22] Wang X, Tang J, Yung K. Optimization of the multi-objective dynamic cell formation problem using a scatter search approach. Int J Adv Manuf Technol 2009:318-29.

[23] Deljoo V, Mirzapour Al-e-hashem SMJ, Deljoo F, Aryanezhad MB. Using genetic algorithm to solve dynamic cell formation problem. Appl Math Model 2010;34:1078-92.

[24] Mahdavi I, Aalaei A, Paydar MM, Solimanpur M. Designing a mathematical model for dynamic cellular manufacturing systems considering production planning and worker assignment. Comput Math with Appl 2010;60:1014-25.

[25] Javadian N, Aghajani A, Rezaeian J, Ghaneian Sebdani M. A multi-objective integrated cellular manufacturing systems design with dynamic system reconfiguration. Int J Adv Manuf Technol 2011;56:307-17.

[26] Rafiee K, Rabbani M, Rafiei H, Rahimi-Vahed A. A new approach towards integrated cell formation and inventory lot sizing in an unreliable cellular manufacturing system. Appl Math Model 2011;35:1810-9.

[27] Saxena L, Jain P. Dynamic cellular manufacturing systems design—a comprehensive model. Int J Adv Manuf Technol 2011;53:11-34.

[28] Kia R, Baboli A, Javadian N, Tavakkoli-moghaddam R, Kazemi M, Khorrami J. Solving a group layout design model of a dynamic cellular manufacturing system with alternative process routings, lot splitting and flexible reconfiguration by simulated annealing. Comput Oper Res 2012;39:2642-58.

[29] Kia R, Khaksar-Haghani F, Javadian N, Tavakkoli-Moghaddam R. Solving a multi-floor layout design model of a dynamic cellular manufacturing system by an efficient genetic algorithm. $\mathrm{J}$ Manuf Syst 2014;33:218-32. doi:10.1016/j.jmsy.2013.12.005.

[30] Rafiei H, Ghodsi R. A bi-objective mathematical model toward dynamic cell formation considering labor utilization. Appl Math Model 2013;37:2308-16.

[31] Kia R, Shirazi H, Javadian N, Tavakkoli-Moghaddam R. Designing group layout of unequalarea facilities in a dynamic cellular manufacturing system with variability in number and shape of cells. Int J Prod Res 2014;53:3390-418. doi:10.1080/00207543.2014.986295. 
[32] Majazi Dalfard V. New mathematical model for problem of dynamic cell formation based on number and average length of intra and intercellular movements. Appl Math Model 2013;37:1884-96.

[33] Shiyas CR, Madhusudanan Pillai V. A mathematical programming model for manufacturing cell formation to develop multiple configurations. J Manuf Syst 2014;33:149-58. doi:10.1016/j.jmsy.2013.10.002.

[34] Deep K, Singh PK. Design of robust cellular manufacturing system for dynamic part population considering multiple processing routes using genetic algorithm. J Manuf Syst 2015;35:155-63. doi:10.1016/j.jmsy.2014.09.008.

[35] Niakan F, Baboli A, Moyaux T, Botta-Genoulaz V. A Multi-objective Mathematical Model Considering Economic and Social Criteria in Dynamic Cell Formation. vol. 439. Berlin, Heidelberg: Springer Berlin Heidelberg; 2014. doi:10.1007/978-3-662-44736-9.

[36] Dehghanian F, Mansour S. Designing sustainable recovery network of end-of-life products using genetic algorithm. Resour Conserv Recycl 2009;53:559-70.

[37] Ramanathan R, Ganesh LS. Group preference aggregation methods employed in AHP: An evaluation and an intrinsic process for deriving members' weightages. Eur J Oper Res 1994;79:249-65. doi:10.1016/0377-2217(94)90356-5.

[38] Leigh JP, Miller TR. Job-related diseases and occupations within a large workers' compensation data set. Am J Ind Med 1998;33:197-211.

[39] Daniell WE, Fulton-Kehoe D, Smith-Weller T, Franklin GM. Occupational hearing loss in Washington state, 1984-1991: II. Morbidity and associated costs. Am J Ind Med 1998;33:529_ 36.

[40] Niebel B, Freivalds A. Niebel's Methods, Standards and Work Design. 12th ed. McGraw-Hill, New York.; 2009.

[41] Nanthavanij S. Analytical Approach for Workplace Noise Assessment. Thammasat Int J Sci Technol 2002;7:8-16.

[42] Sanders MS, McCormick EJ. Human factors in engineering and design. vol. 1993. 1993.

[43] Tharmmaphornphilas W. Developing worker rotation schedule based upon workers skills to minimize occupation injury. 35th Int. Conf. Comput. Ind. Eng. Istanbul, Turkey., 2002.

[44] Aryanezhad MB, Kheirkhah AS, Deljoo V, Mirzapour Al-e-hashem SMJ. Designing safe job rotation schedules based upon workers’ skills. Int J Adv Manuf Technol 2008;41:193-9.

[45] National Institute of Occupational Safety and Healt (NIOSH). The National Occupational Research Agenda (NORA). Dep Heal Hum Serv Washington, DC 1996:96-115.

[46] Occupational Safety and Health Administration (OSHA). Guidelines for noise enforcement: appendix A. US Dep Labor, Washington, DCOccupational Saf Heal Adm 1983:2-2.35A.

[47] National Institute of Occupational Safety and Healt (NIOSH). Criteria for a recommended standard: occupational noise exposure. Dep Heal Hum Serv Washington, DC 1998:98-126. 
[48] Garey MR, Johnson DS. Computers and Intractability: A Guide to the Theory of NPCompleteness (Series of Books in the Mathematical Sciences). W. H. Freeman; 1979.

[49] Deb K, Pratap A, Agarwal S, Meyarivan T. A fast and elitist multiobjective genetic algorithm: NSGA-II. Evol Comput IEEE Trans 2002;6:182-97.

[50] Smith KI, Everson RM, Fieldsend JE. Dominance measures for multi-objective simulated annealing. Proc. 2004 Congr. Evol. Comput. (IEEE Cat. No.04TH8753), vol. 1, IEEE; n.d., p. 23-30. doi:10.1109/CEC.2004.1330833.

[51] Tavakkoli-Moghaddam R, Azarkish M, Sadeghnejad-Barkousaraie A. A new hybrid multiobjective Pareto archive PSO algorithm for a bi-objective job shop scheduling problem. Expert Syst Appl 2011;38:10812-21.

[52] Mohammadi M, Jolai F, Tavakkoli-Moghaddam R. Solving a new stochastic multi-mode p-hub covering location problem considering risk by a novel multi-objective algorithm. Appl Math Model 2013;37:10053-73. doi:10.1016/j.apm.2013.05.063. 